\title{
Analytical Dispersion Equations of a Lossy Coaxial Waveguide in the Microwave and Visible Spectra
}

\author{
Yong Heui Cho"
}

\begin{abstract}
Analytical hybrid-mode dispersion relations of a lossy coaxial waveguide were rigorously analyzed using a mode-matching technique. In order to model a practical coaxial line with inevitable losses, we adopted an all-dielectric coaxial waveguide surrounded by the perfect electric conductor (PEC) boundary. The rigorous dispersion characteristics of the TM $\mathrm{TM}_{01}, \mathrm{TE}_{01}$, and $\mathrm{EH}_{11}$ modes were investigated for lossy coaxial waveguides filled with different electrical conductivities. Based on the exact solutions, approximate but accurate dispersion equations were proposed for the $\mathrm{TM}_{0 p}, \mathrm{TE}_{0 p}, \mathrm{EH}_{m p}$, and $\mathrm{HE}_{m p}$ modes in order to estimate and compare the behaviors of complex propagation constants in the microwave and visible spectra.
\end{abstract}

Key Words: Coaxial Line, Complex Propagation Constant, Dispersion Relation, Guided Wave, Mode-Matching Technique.

\section{INTRODUCTION}

A coaxial waveguide is a fundamental transmission line to stably guide a light for optoelectronics [1-3] or precisely measure the reflection coefficient and impedance up to millimeter-wave bands $[4,5]$. Accurate evaluation of wave propagation through a practical coaxial waveguide is essential for quantifying measurement uncertainty occurring in microwave transmission [5]. Even in the visible spectrum, a nanocoax [3] is one of the promising structures for low-loss propagation composed of plasmonic and photonic modes, where the permittivity can be negative or complex to model real metals at optical frequency.

Since a practical coaxial line is inherently lossy owing to finite electrical conductivity, the TM wave $\left(E_{z} \neq 0, H_{z}=0\right)$ instead of the TEM wave $\left(E_{z}=0, H_{z}=0\right)$ propagates and is gradually decreased in the $z$-direction. This behavior makes the dispersion analysis of the lossy coaxial waveguide more involved. Therefore, it is of great importance to derive and formulate an exact and rigorous dispersion relation of the lossy coaxial waveguide, which can be modeled as a multilayered dielectric coaxial waveguide [1, 6-8]. A dielectric coaxial waveguide was analyzed using the boundary conditions of an open region [6] and an infinitely lossy outer conductor $[7,8]$. In the following sections, we apply a standard mode-matching technique [9] for analyzing a dielectric coaxial waveguide surrounded by the perfect electric conductor (PEC) boundary. A newly-derived rigorous dispersion relation is used to determine precise complex propagation constants for the hybrid, TM, and TE modes in microwave and optical spectra, where we use Davidenko's method $[10,11]$ for searching complex roots.

Using the conductor condition or high permittivity approximation, we obtained simplified but accurate dispersion equations useful for characterizing the transmission behaviors of the lossy coaxial waveguide. This means that our rigorous dispersion equations can be utilized to generate the field distributions for the $\mathrm{TM}_{0 p}, \mathrm{TE}_{0 p}, \mathrm{EH}_{m p}$, and $\mathrm{HE}_{m p}$ modes and formulate the

Manuscript received August 22, 2020 ; Revised November 5, 2020 ; Accepted December 1, 2020. (ID No. 20200822-125J)

School of Information and Communication Engineering, Mokwon University, Daejeon, Korea.

"Corresponding Author: Yong Heui Cho (e-mail: yongheui.cho@gmail.com)

This is an Open-Access article distributed under the terms of the Creative Commons Attribution Non-Commercial License (http://creativecommons.org/licenses/by-nc/4.0) which permits unrestricted non-commercial use, distribution, and reproduction in any medium, provided the original work is properly cited.

(c) Copyright The Korean Institute of Electromagnetic Engineering and Science. All Rights Reserved. 
scattering characteristics of canonical coaxial structures used in a coaxial calibration kit [5]. The quasi-TEM mode also yields a closed-form approximate solution for the $\mathrm{TM}_{01}$-mode complex propagation constant, which becomes identical to the propagation constant $[5,7]$ in the high electrical conductivity limit.

\section{Mode-MATChING ANALYSIS}

A lossy coaxial waveguide can be modeled using a multilayered dielectric coaxial waveguide $[1,6-8]$ as shown in Fig. 1 , where the medium constants- $\epsilon_{1}, \epsilon_{2}, \epsilon_{3}$ and $\mu_{1}, \mu_{2}, \mu_{3}-$ can be any complex number. We use and omit $e^{i(\beta z-\omega t)}$ for the time convention, where $\beta$ is a complex propagation constant composed of phase $(\mathfrak{R}[\beta])$ and attenuation $(\mathfrak{I}[\beta])$ constants. The interesting point of the geometry shown in Fig. 1 is that a dielectric coaxial waveguide $\left[1,6^{-8}\right]$ shown in Fig. 2 is entirely surrounded by the PEC boundary. It is known that unwanted multiple leaky modes [11] are inevitably generated in open magnetodielectric waveguides including dielectric coaxial structures $[1,6]$ with an open boundary as shown in Fig. 2. The existence of leaky phenomena caused by the open boundary makes a dispersion analysis of the dielectric coaxial waveguide much more involved. For instance, the dielectric coaxial waveguide shown in Fig. 2 should satisfy a leaky dispersion relation in an open region as

$$
k_{0}^{2}=\kappa_{\rho}^{2}+\beta^{2},
$$

where $k_{0}=\omega \sqrt{\mu_{0} \epsilon_{0}}$, and $\kappa_{\rho}$ is a leaky wavenumber for a radial direction $\rho=\sqrt{x^{2}+y^{2}}$. Equating the imaginary parts of the left and right sides of Eq. (1) yields a leaky-wave relation for $\kappa_{\rho}$ and $\beta$ as

$$
\Re\left[\kappa_{\rho}\right] \mathfrak{I}\left[\kappa_{\rho}\right]=-\mathfrak{R}[\beta] \Im[\beta],
$$

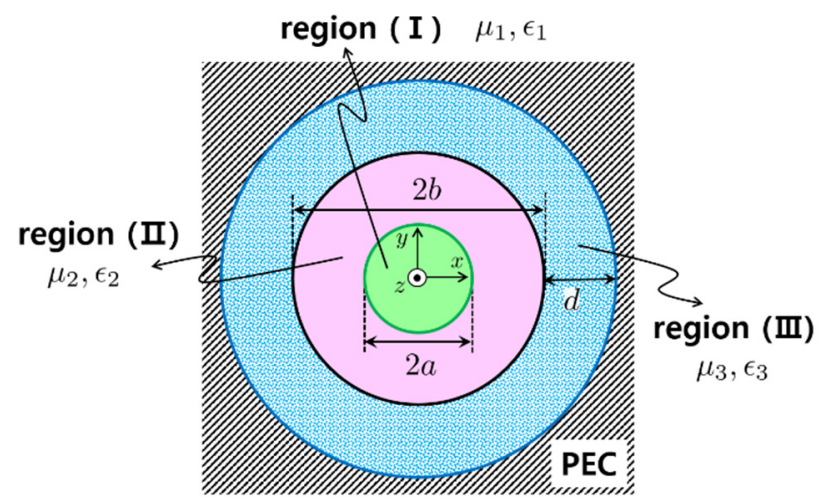

Fig. 1. Modeling of a lossy coaxial waveguide using a multilayered dielectric coaxial waveguide surrounded by the perfect electric conductor (PEC) boundary.

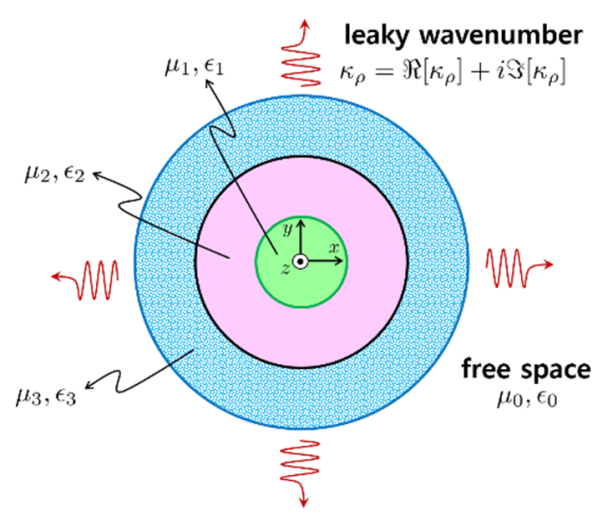

Fig. 2. The geometry of a three-layered dielectric coaxial waveguide placed in free space or with an open boundary.

where the phase term of a guided wave is $e^{i\left(\kappa_{\rho} \rho+\beta z\right)}$. Eq. (2) indicates that the radial and longitudinal radiation conditions of $\kappa_{\rho}$ and $\beta$ cannot be satisfied at the same time, owing to the fact that the signs of the real or imaginary parts of $\kappa_{\rho}$ and $\beta$ are always different from each other. Contrary to the geometry shown in Fig. 2, the PEC boundary and lossy region (III) introduced in Fig. 1 enable us to block the leaky modes completely and model the conductor loss precisely. This is because the PEC boundary at $\rho=b+d$ is imposed and $\epsilon_{3}$ can be complex, thus confirming there are no leaky modes and the waves are evanescent in region (III). Therefore, the field representations for regions (I) through (III) as shown in Fig. 1 are formulated using magnetic and electric vector potentials [9]:

$$
\begin{gathered}
A_{Z}^{\mathrm{I}}(\bar{r})=\sum_{m=-\infty}^{\infty} A_{m} J_{m}\left(\kappa_{1} \rho\right) e^{i m \phi}, \\
A_{Z}^{\mathrm{II}}(\bar{r})=\sum_{m=-\infty}^{\infty}\left[E_{m}^{(1)} J_{m}\left(\kappa_{2} \rho\right)+E_{m}^{(2)} N_{m}\left(\kappa_{2} \rho\right)\right] e^{i m \phi}, \\
A_{Z}^{\mathrm{III}}(\bar{r})=\sum_{m=-\infty}^{\infty} G_{m} C_{m}\left(\kappa_{3} \rho\right) e^{i m \phi}, \\
F_{Z}^{\mathrm{I}}(\bar{r})=\sum_{m=-\infty}^{\infty} B_{m} J_{m}\left(\kappa_{1} \rho\right) e^{i m \phi}, \\
F_{Z}^{\mathrm{II}}(\bar{r})=\sum_{m=-\infty}^{\infty}\left[F_{m}^{(1)} J_{m}\left(\kappa_{2} \rho\right)+F_{m}^{(2)} N_{m}\left(\kappa_{2} \rho\right)\right] e^{i m \phi}, \\
F_{Z}^{\mathrm{III}}(\bar{r})=\sum_{m=-\infty}^{\infty} H_{m} D_{m}\left(\kappa_{3} \rho\right) e^{i m \phi},
\end{gathered}
$$

where $A_{m}, B_{m}, E_{m}^{(1),(2)}, F_{m}^{(1),(2)}, G_{m}$, and $H_{m}$ are the unknown modal coefficients for the $m$ th azimuthal mode, $\rho=$ $\sqrt{x^{2}+y^{2}}, \phi=\tan ^{-1}(y / x), \quad \kappa_{n}=\sqrt{k_{n}^{2}-\beta^{2}}, \quad k_{n}=$ $\omega \sqrt{\mu_{n} \epsilon_{n}}$ 


$$
\begin{aligned}
& C_{m}(\psi)=J_{m}(\psi) N_{m}\left[\kappa_{3}(b+d)\right] \\
& -N_{m}(\psi) J_{m}\left[\kappa_{3}(b+d)\right], \\
& D_{m}(\psi)=J_{m}(\psi) N_{m}^{\prime}\left[\kappa_{3}(b+d)\right] \\
& -N_{m}(\psi) J_{m}^{\prime}\left[\kappa_{3}(b+d)\right],
\end{aligned}
$$

and $(\cdot)^{\prime}$ denotes differentiation for the entire argument; $J_{m}(\cdot)$ and $N_{m}(\cdot)$ are the $m$ th order Bessel functions of the first and second kinds, respectively. Based on the standard mode-matching analysis [9], we enforce $E_{z^{-}}, E_{\phi^{-}}, H_{z^{-}}$, and $H_{\phi}$-field continuities at $\rho=a$ and $b$ for the $m$ th azimuthal mode. First, by multiplying the $E_{z^{-}}$and $H_{z}$-field continuities at $\rho=a$ by $e^{-i l \phi}(l=0, \pm 1, \pm 2, \cdots)$ and integrating over $0 \leq \phi \leq 2 \pi$ yields, respectively, we get:

$$
\begin{aligned}
& A_{m} J_{m}\left(u_{1}\right)=\frac{\mu_{1} \epsilon_{1} \kappa_{2}^{2}}{\mu_{2} \epsilon_{2} \kappa_{1}^{2}} \varepsilon_{m}\left(u_{2}\right), \\
& B_{m} J_{m}\left(u_{1}\right)=\frac{\mu_{1} \epsilon_{1} \kappa_{2}^{2}}{\mu_{2} \epsilon_{2} \kappa_{1}^{2}} \varphi_{m}\left(u_{2}\right),
\end{aligned}
$$

where $u_{n}=\kappa_{n} a$,

$$
\begin{aligned}
& \varepsilon_{m}(u)=E_{m}^{(1)} J_{m}(u)+E_{m}^{(2)} N_{m}(u), \\
& \varphi_{m}(u)=F_{m}^{(1)} J_{m}(u)+F_{m}^{(2)} N_{m}(u) .
\end{aligned}
$$

Next, we apply and integrate the $E_{\phi^{-}}$and the $H_{\phi}$-field continuities at $\rho=a$ with $e^{-i l \phi}$ to obtain the additional field-matching equations, respectively:

$$
\begin{aligned}
& \frac{i m}{Z_{1}^{\mathrm{TE}}} A_{m} J_{m}\left(u_{1}\right)-u_{1} B_{m} J_{m}^{\prime}\left(u_{1}\right)= \\
& \frac{\epsilon_{1}}{\epsilon_{2}}\left[\frac{i m}{Z_{2}^{\mathrm{TE}}} \varepsilon_{m}\left(u_{2}\right)-u_{2} \varphi_{m}^{\prime}\left(u_{2}\right)\right], \\
& u_{1} A_{m} J_{m}^{\prime}\left(u_{1}\right)+\frac{i m}{Y_{1}^{\mathrm{TM}}} B_{m} J_{m}\left(u_{1}\right)= \\
& \frac{\mu_{1}}{\mu_{2}}\left[u_{2} \varepsilon_{m}^{\prime}\left(u_{2}\right)+\frac{i m}{Y_{2}^{\mathrm{TM}}} \varphi_{m}\left(u_{2}\right)\right],
\end{aligned}
$$

where $Z_{n}^{\mathrm{TE}}=\omega \mu_{n} /_{\beta}, \quad Y_{n}^{\mathrm{TM}}=\omega \epsilon_{n} /_{\beta}, \quad \varepsilon_{m}^{\prime}(u)=\frac{d}{d u} \varepsilon_{m}(u)$, and $\varphi_{m}^{\prime}(u)=\frac{d}{d u} \varphi_{m}(u)$. Similar to Eq. (11), in Eqs. (12), (15), and (16), we utilize the tangential boundary conditions for the $E_{z^{-}}, E_{\phi^{-}}, H_{z^{-}}$, and $H_{\phi^{-}}$fields at $\rho=b$. Then, we formulate the modal relations of $E_{m}^{(1),(2)}, F_{m}^{(1),(2)}, G_{m}$ and $H_{m}$ as

$$
\begin{aligned}
& G_{m} C_{m}\left(v_{3}\right)=\frac{\mu_{3} \epsilon_{3} \kappa_{2}^{2}}{\mu_{2} \epsilon_{2} \kappa_{3}^{2}} \varepsilon_{m}\left(v_{2}\right), \\
& H_{m} D_{m}\left(v_{3}\right)=\frac{\mu_{3} \epsilon_{3} \kappa_{2}^{2}}{\mu_{2} \epsilon_{2} \kappa_{3}^{2}} \varphi_{m}\left(v_{2}\right),
\end{aligned}
$$

$$
\begin{gathered}
\frac{i m}{Z_{3}^{\mathrm{TE}}} G_{m} C_{m}\left(v_{3}\right)-v_{3} H_{m} D_{m}^{\prime}\left(v_{3}\right)= \\
\frac{\epsilon_{3}}{\epsilon_{2}}\left[\frac{i m}{Z_{2}^{\mathrm{TE}}} \varepsilon_{m}\left(v_{2}\right)-v_{2} \varphi_{m}^{\prime}\left(v_{2}\right)\right], \\
v_{3} G_{m} C_{m}^{\prime}\left(v_{3}\right)+\frac{i m}{Y_{3}^{\mathrm{TM}}} H_{m} D_{m}\left(v_{3}\right)= \\
\frac{\mu_{3}}{\mu_{2}}\left[v_{2} \varepsilon_{m}^{\prime}\left(v_{2}\right)+\frac{i m}{Y_{2}^{\mathrm{TM}}} \varphi_{m}\left(v_{2}\right)\right],
\end{gathered}
$$

where $v_{n}=\kappa_{n} b$. Combining and simplifying Eqs. (11), (12), and (15)-(20), we obtained the $m$ th hybrid-mode dispersion relation as

$$
\left|\boldsymbol{\Phi}_{m}(\beta)\right|=0,
$$

where $|\mathbf{A}|$ is the determinant of a matrix $\mathbf{A}$ and a system of simultaneous equations for $E_{m}^{(1),(2)}, F_{m}^{(1),(2)}$ is given by:

$$
\begin{gathered}
\boldsymbol{\Phi}_{m}(\beta) \mathbf{E}_{m}= \\
{\left[\begin{array}{cccc}
\phi_{11}^{(1)} & \phi_{11}^{(2)} & \phi_{12}^{(1)} & \phi_{12}^{(2)} \\
\phi_{21}^{(1)} & \phi_{21}^{(2)} & \phi_{11}^{(1)} & \phi_{11}^{(2)} \\
\phi_{31}^{(1)} & \phi_{31}^{(2)} & \phi_{32}^{(1)} & \phi_{32}^{(2)} \\
\phi_{41}^{(1)} & \phi_{41}^{(2)} & \phi_{31}^{(1)} & \phi_{31}^{(2)}
\end{array}\right]\left[\begin{array}{c}
E_{m}^{(1)} \\
E_{m}^{(2)} \\
F_{m}^{(1)} \\
F_{m}^{(2)}
\end{array}\right]=0 .}
\end{gathered}
$$

The elements of $\boldsymbol{\Phi}_{m}(\beta)$ are defined by:

$$
\phi_{11}^{(n)}=i m \frac{u_{2}^{2}-u_{1}^{2}}{\left(u_{1} u_{2}\right)^{2}} Z_{m}^{(n)}\left(u_{2}\right)
$$

$$
\begin{aligned}
\phi_{12}^{(n)}= & Z_{2}^{\mathrm{TE}} \frac{Z_{m}^{(n) \prime}\left(u_{2}\right)}{u_{2}}-Z_{1}^{\mathrm{TE}} \frac{J_{m}^{\prime}\left(u_{1}\right)}{J_{m}\left(u_{1}\right)} \frac{Z_{m}^{(n)}\left(u_{2}\right)}{u_{1}}, \\
\phi_{21}^{(n)}= & Y_{1}^{\mathrm{TM}} \frac{J_{m}^{\prime}\left(u_{1}\right)}{J_{m}\left(u_{1}\right)} \frac{Z_{m}^{(n)}\left(u_{2}\right)}{u_{1}}-Y_{2}^{\mathrm{TM}} \frac{Z_{m}^{(n) \prime}\left(u_{2}\right)}{u_{2}}, \\
& \phi_{31}^{(n)}=i m \frac{v_{2}^{2}-v_{3}^{2}}{\left(v_{1} v_{3}\right)^{2}} Z_{m}^{(n)}\left(v_{2}\right), \\
\phi_{32}^{(n)}= & Z_{2}^{\mathrm{TE}} \frac{Z_{m}^{(n) \prime}\left(v_{2}\right)}{v_{2}}-Z_{3}^{\mathrm{TE}} \frac{D_{m}^{\prime}\left(v_{3}\right)}{D_{m}\left(v_{3}\right)} \frac{Z_{m}^{(n)}\left(v_{2}\right)}{v_{3}},
\end{aligned}
$$

$$
\phi_{41}^{(n)}=Y_{3}^{\mathrm{TM}} \frac{C_{m}^{\prime}\left(v_{3}\right)}{C_{m}\left(v_{3}\right)} \frac{Z_{m}^{(n)}\left(v_{2}\right)}{v_{3}}-Y_{2}^{\mathrm{TM}} \frac{Z_{m}^{(n) \prime}\left(v_{2}\right)}{v_{2}},
$$

where

$$
Z_{m}^{(n)}(u)=\left\{\begin{array}{ll}
J_{m}(u) & \text { if } n=1 \\
N_{m}(u) & \text { if } n=2
\end{array} .\right.
$$

Therefore, a complex propagation constant $\beta$ can be determined by solving Eq. (21). When $m=0$, the hybridmode dispersion relation shown in Eq. (21) can be divided into 


$$
\begin{aligned}
\underbrace{\left(\phi_{21}^{(1)} \phi_{41}^{(2)}-\phi_{21}^{(2)} \phi_{41}^{(1)}\right)}_{\mathrm{TM}_{0 p} \text { mode }} & \times \underbrace{\left(\phi_{12}^{(1)} \phi_{32}^{(2)}-\phi_{12}^{(2)} \phi_{32}^{(1)}\right)}_{\mathrm{TE}_{0 p} \operatorname{mode}} \\
= & 0 .
\end{aligned}
$$

Eq. (30) clearly proves that the TM and TE modes exist for $m=0$, although the coaxial waveguide is filled with lossy dielectrics. Since Eq. (21) is applicable to a general coaxial waveguide of any $\mu_{n}$ and $\epsilon_{n}$, we can use Eq. (21) to analyze canonical waveguides, including the PEC circular and coaxial waveguides. For instance, when $\mu_{n}=\mu_{0}$ and $\epsilon_{n}=\epsilon_{0}$, the geometry shown in Fig. 1 becomes a PEC circular waveguide with a radius of $b+d$, and Eq. (21) is thus simplified to

$$
\underbrace{\phi_{41}^{(1)}}_{\text {TM mode }} \times \underbrace{\phi_{32}^{(1)}}_{\text {TE mode }}=0
$$

Similarly, when $\mu_{n}=\mu_{0}, \epsilon_{1} \rightarrow \infty$, and $\epsilon_{3}=\epsilon_{2}$, the geometry in Fig. 1 is considered a PEC coaxial waveguide. Then, the dispersion equation in Eq. (21) reduces to that of a PEC coaxial waveguide as

$$
\begin{aligned}
\underbrace{\left(\phi_{11}^{(1)} \phi_{41}^{(2)}-\phi_{11}^{(2)} \phi_{41}^{(1)}\right)}_{\text {TM mode }} & \times \underbrace{\left(\phi_{12}^{(1)} \phi_{32}^{(2)}-\phi_{12}^{(2)} \phi_{32}^{(1)}\right)}_{\text {TE mode }} \\
= & 0 .
\end{aligned}
$$

\section{NUMERICAL COMPUTATIONS}

We used Davidenko's method $[10,11]$ to search a complex root $\beta$ by equating $\left|\boldsymbol{\Phi}_{m}(\beta)\right|=0$ as shown in Eq. (21). Davidenko's method in [11] uses the fourth-order Runge-Kutta method and the Newton-Raphson method in the complex domain, which is suitable for searching the complex propagation constant $\beta$. An iterative update equation for the next approximate solution $\beta_{n+1}$ is given by

$$
\beta_{n+1}=\beta_{n}+\Delta \beta_{n+1}
$$

where $\beta_{n}$ is the current approximate value for $\beta$ and $\Delta \beta_{n+1}$ is defined in [11, Eq. (10)]. The next differential step $\Delta \beta_{n+1}$ is computed using $\beta_{n}, \Delta \beta_{n}$, and $h$, where $\beta_{0}$ and $\Delta \beta_{0}$ are the initial value and step for root searching, respectively, and $h$ is a fixed step of the Runge-Kutta method. As $n$ increases very steeply, $\beta_{n+1}$ stably converges to $\beta[10,11]$. We set $h=1$ and $\Delta \beta_{0}=\beta_{0} / 100$ for all root-searching computations.

The $\mathrm{TM}_{01}$-mode dispersion results of the lossy coaxial waveguide are shown in Fig. 3 using $m=0$ and $f=100$ $\mathrm{GHz}$, where the $\mathrm{TM}_{01}$ mode $(m=0)$ is a dominant quasiTEM mode. We assume that regions (I) and (III) are filled with lossy conductors using $\epsilon_{1}=\epsilon_{3}=\epsilon_{0}\left(1+i \frac{\sigma}{\omega \epsilon_{0}}\right)$, where $\sigma$ denotes the electrical conductivity of a lossy dielectric. Using Eq. (30) and the conductor condition $\left(\left|\epsilon_{1}\right| \gg 1\right.$,

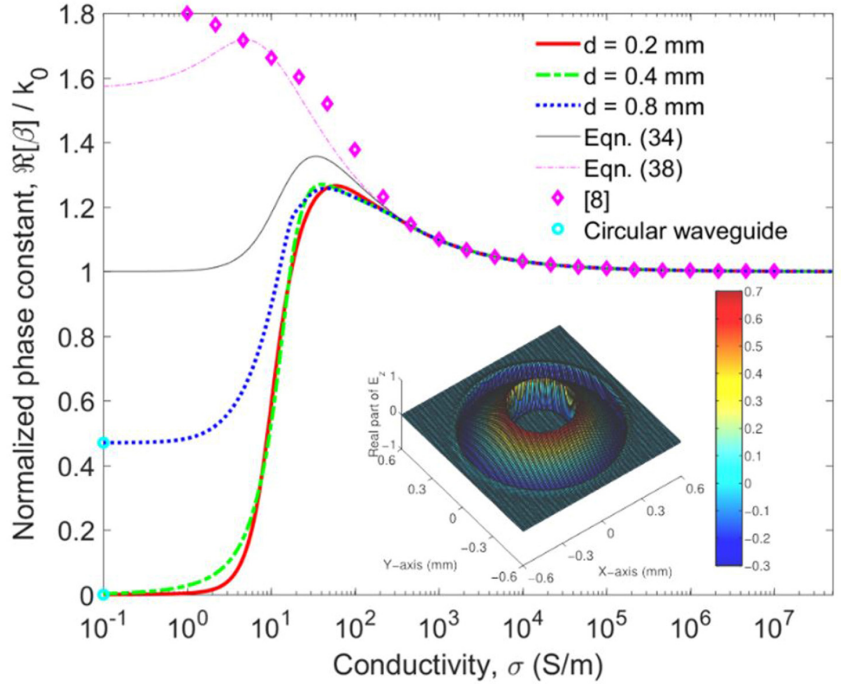

(a)

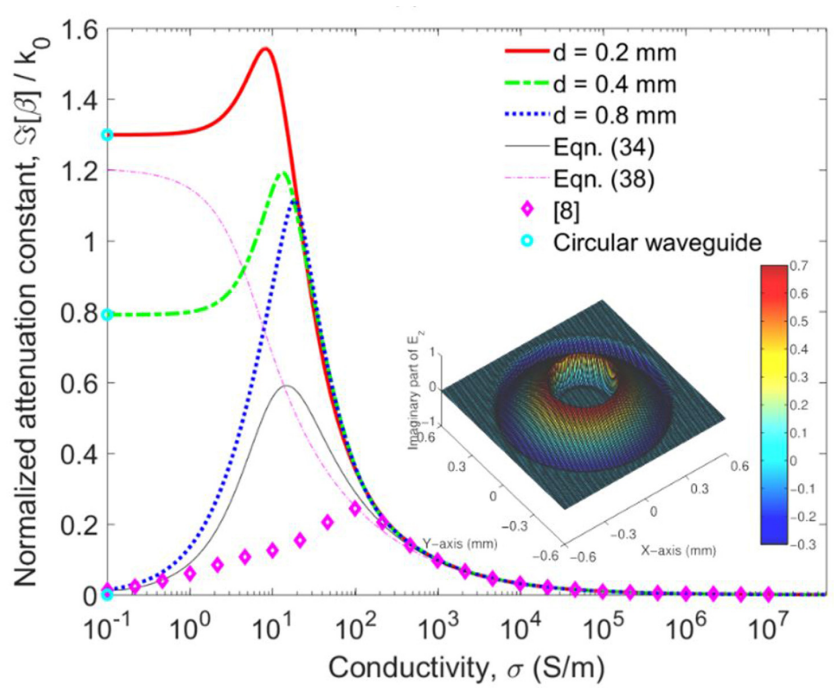

(b)

Fig. 3. Complex dispersion relation versus electrical conductivity $\sigma$ for the $\mathrm{TM}_{01}$ mode in the lossy coaxial waveguide using $m=0, f=100 \mathrm{GHz}, 2 a=0.434 \mathrm{~mm}$, $2 b=1 \mathrm{~mm}, \mu_{n}=\mu_{0}, \epsilon_{1}=\epsilon_{3}=\epsilon_{0}\left(1+i \frac{\sigma}{\omega \epsilon_{0}}\right)$, and $\epsilon_{2}=\epsilon_{0}$ : (a) phase constant $\Re[\beta]$ and (b) attenuation constant $\Im[\beta]$.

$\left.\left|\epsilon_{3}\right| \gg 1\right)$, we obtain an approximate dispersion relation for the $\mathrm{TM}_{0 p}$ mode as

$$
i U_{0}\left(v_{2}\right)-P_{3} U_{0}^{\prime}\left(v_{2}\right)+P_{1} V_{0}\left(v_{2}\right) \approx 0,
$$

where $P_{n}=\epsilon_{2} \kappa_{n} / \epsilon_{n} \kappa_{2}$,

$$
\begin{gathered}
U_{m}(v)=J_{m}\left(u_{2}\right) N_{m}(v)-N_{m}\left(u_{2}\right) J_{m}(v), \\
V_{m}(v)=J_{m}^{\prime}\left(u_{2}\right) N_{m}(v)-N_{m}^{\prime}\left(u_{2}\right) J_{m}(v) .
\end{gathered}
$$

Note that setting $U_{m}\left(v_{2}\right)$ and $V_{m}^{\prime}\left(v_{2}\right)$ equal to zero yields 
the $\mathrm{TM}_{m p^{-}}$and $\mathrm{TE}_{m p}$-mode dispersion relations of an ideal coaxial line, respectively, where the subscript $m p$ means that $m$ and $p$ are the numbers of field variations in the $\phi$ and $\rho$-directions, respectively. This property indicates that Eq. (34) is a perturbed but accurate solution for the lossy coaxial waveguide when $P_{1}$ and $P_{3}$ are very small owing to $\left|\epsilon_{1}\right| \gg 1$ and $\left|\epsilon_{3}\right| \gg 1$. Utilizing $\beta \approx k_{2},\left|k_{1}\right| \gg|\beta|$, and $\left|k_{3}\right| \gg|\beta|$, Eq. (34) can be further simplified to

$$
\kappa_{2} \approx i \epsilon_{2} \frac{\frac{\kappa_{1}}{\epsilon_{1}} V_{0}\left(v_{2}\right)-\frac{\kappa_{3}}{\epsilon_{3}} U_{0}^{\prime}\left(v_{2}\right)}{U_{0}\left(v_{2}\right)} \approx \frac{i k_{2}^{2}}{\mu_{2}} \frac{\frac{\mu_{1}}{k_{1}} N_{0}^{\prime}\left(u_{2}\right)+\frac{\mu_{3}}{k_{3}} N_{0}^{\prime}\left(v_{2}\right)}{N_{0}\left(u_{2}\right)-N_{0}\left(v_{2}\right)},
$$

when $u \rightarrow 0, N_{0}(u)$ and $N_{0}^{\prime}(u)$ become $\frac{2}{\pi} \ln \left(\frac{u}{2}\right)$ and $\frac{2}{\pi u}$, respectively. Therefore, an approximate closed-form solution for Eq. (37) is finally obtained as

$$
\beta \approx k_{2} \sqrt{1+\frac{i}{\mu_{2} \ln \frac{b}{a}}\left(\frac{\mu_{1}}{k_{1} a}+\frac{\mu_{3}}{k_{3} b}\right)} .
$$

Note that Eq. (38) is identical to [7, Eq. (37)] even though the boundary conditions for the outer conductors are different from each other. When $\mu_{n}=\mu_{0}$ and $\sigma \gg 1$, Eq. (38) becomes a complex propagation constant [5] obtained by the transmission line theory and penetration depth $\delta_{s}$. Fig. 3 clearly indicates that the thickness $(d)$ of an outer conductor hardly affects complex dispersion relations for $\sigma \geq 10^{3} \mathrm{~S} / \mathrm{m}$ and $d \geq 0.2 \mathrm{~mm} \approx 4 \delta_{s}$, where $\delta_{s} \approx 50 \mu \mathrm{m}$ and $\sigma=10^{3}$ $\mathrm{S} / \mathrm{m}$. A comparison with [8] provides a favorable agreement when $\sigma \geq 10^{3} \mathrm{~S} / \mathrm{m}$. This is because [8] assumes an infinite outer conductor $(d \rightarrow \infty)$ and the effects of the thick outer conductor are negligible for high electrical conductivity, where the outer conductor shown in Fig. 1 has finite thickness $(d)$, which differs from [8]. As is also shown in Fig. 3, Eqs. (34) and (38) are practically good formulas in lieu of Eq. (21) for $\sigma \geq 10^{3} \mathrm{~S} / \mathrm{m}$. When $\sigma$ approaches zero, Eq. (31) predicts that $\beta$ becomes that of a PEC circular waveguide denoted as $\bigcirc$ in Fig. 3. The insets in Fig. 3 illustrate the normalized real and imaginary parts of the $E_{z}$-field distributions when $\sigma=10^{4} \mathrm{~S} / \mathrm{m}$ and $\beta \approx 2161.77+65.96 \mathrm{irad} / \mathrm{m}$. The real and imaginary $E_{z}$-fields of the $\mathrm{TM}_{01}$ mode are continuous across the boundaries at $\rho=a$ and $b$, thus verifying that Davidenko's method in Eq. (33) is effective for searching the complex root $\beta$ of a lossy coaxial waveguide. In addition, the magnitude of the $E_{z}$-fields is rapidly attenuated within lossy dielectrics in regions (I) and (III), and thus their field distributions illustrate the behaviors of the penetration depth very well.

Fig. 4 shows the dispersion behaviors of the $\mathrm{TM}_{01}, \mathrm{TE}_{01}$, and $\mathrm{EH}_{11}$ modes in the lossy coaxial waveguide versus micro-

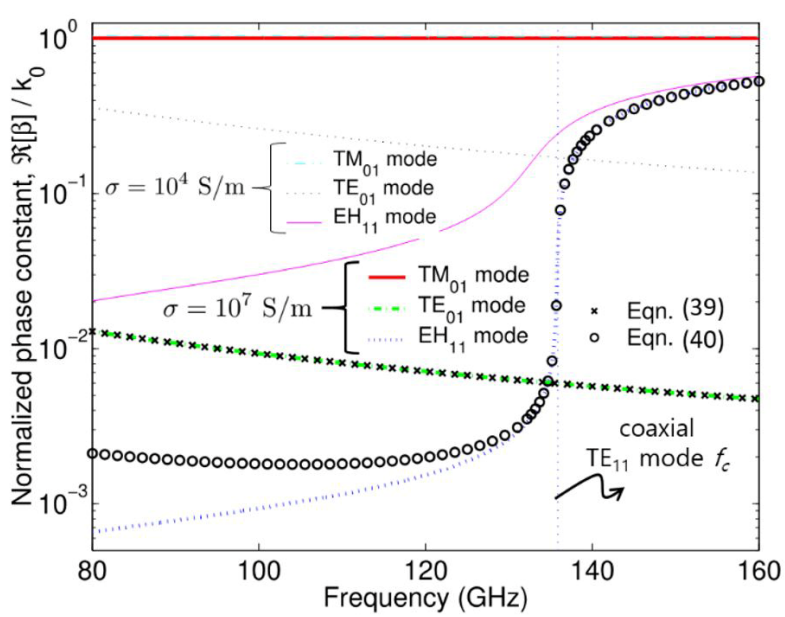

(a)

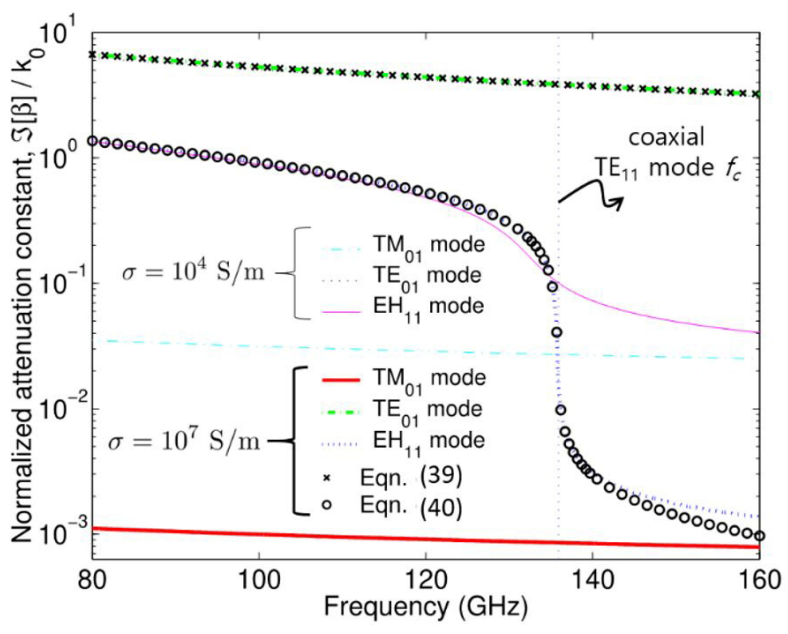

(b)

Fig. 4. Microwave behaviors of complex dispersion relations of multiple modes in the lossy coaxial waveguide using $m=$ 0 or $1, d=0.4 \mathrm{~mm}$, and the same parameters given in the caption of Fig. 3. The coaxial $\mathrm{TE}_{11}$-mode cutoff frequency $\left(f_{c}\right)$ is computed using an ideal coaxial line: (a) phase constant $\mathfrak{R}[\beta]$ and (b) attenuation constant $\mathfrak{I}[\beta]$.

wave frequency. The $\mathrm{TM}_{01}$ mode has the lowest attenuation when compared with the $\mathrm{TE}_{01}$ and $\mathrm{EH}_{11}$ modes, thus indicating that the $\mathrm{TM}_{01}$ mode is a dominant mode irrespective of $\sigma$. Applying Eq. (30) and the conductor condition $\left(\left|\epsilon_{1}\right| \gg 1,\left|\epsilon_{3}\right| \gg 1\right)$ yields an approximate dispersion equation for the $\mathrm{TE}_{0 p}$ mode as

$$
i V_{0}^{\prime}\left(v_{2}\right)+Q_{3} V_{0}\left(v_{2}\right)-Q_{1} U_{0}^{\prime}\left(v_{2}\right) \approx 0,
$$

where $Q_{n}=\mu_{n} \kappa_{2} / \mu_{2} \kappa_{n}$. Similar to Eqs. (34) and (39), the dispersion relation Eq. (21) approximately reduces to

$$
\begin{aligned}
& \underbrace{\left[i U_{m}\left(v_{2}\right)-P_{3} U_{m}^{\prime}\left(v_{2}\right)+P_{1} V_{m}\left(v_{2}\right)\right]}_{\mathrm{HE}_{m p} \text { mode }} \\
& \times \underbrace{\left[i V_{m}^{\prime}\left(v_{2}\right)+Q_{3} V_{m}\left(v_{2}\right)-Q_{1} U_{m}^{\prime}\left(v_{2}\right)\right]}_{\mathrm{EH}_{m p} \text { mode }} \approx 0 .
\end{aligned}
$$


When $\sigma \geq 10^{7} \mathrm{~S} / \mathrm{m}$, the magnitude of $\epsilon_{1}$ and $\epsilon_{3}$ becomes very high and thus the $\mathrm{TE}_{01^{-}}$and $\mathrm{EH}_{11}$-mode $\beta$ computed by Eqs. (39) and (40) agree well with more precise solutions obtained by Eqs. (30) and (21), respectively. In Fig. 4 , the attenuation constant $(\Im[\beta])$ of the $\mathrm{EH}_{11}$ mode rapidly decreases above $f_{c} \approx 135.9 \mathrm{GHz}$. Therefore, the $\mathrm{TM}_{01}$ and $\mathrm{EH}_{11}$ modes coexist and propagate along the lossy coaxial waveguide above $f_{c}$. Considering Eq. (40), the cutoff frequency $\left(f_{c}\right)$ of the lossy $\mathrm{EH}_{11}$ mode can be approximately determined using $V_{1}^{\prime}\left(v_{2}\right) \approx 0$, which is related to $f_{c}$ of the $\mathrm{TE}_{11}$ mode in an ideal coaxial line. The vertical dotted lines as shown in Fig. 4 denote $f_{c}$ of the coaxial $\mathrm{TE}_{11}$ mode.

Fig. 5 illustrates the dispersion characteristics of the $\mathrm{TM}_{01}$ and $\mathrm{EH}_{11}$ modes within the visible spectrum. To theoretically obtain the dispersion relations of the lossy coaxial wave-

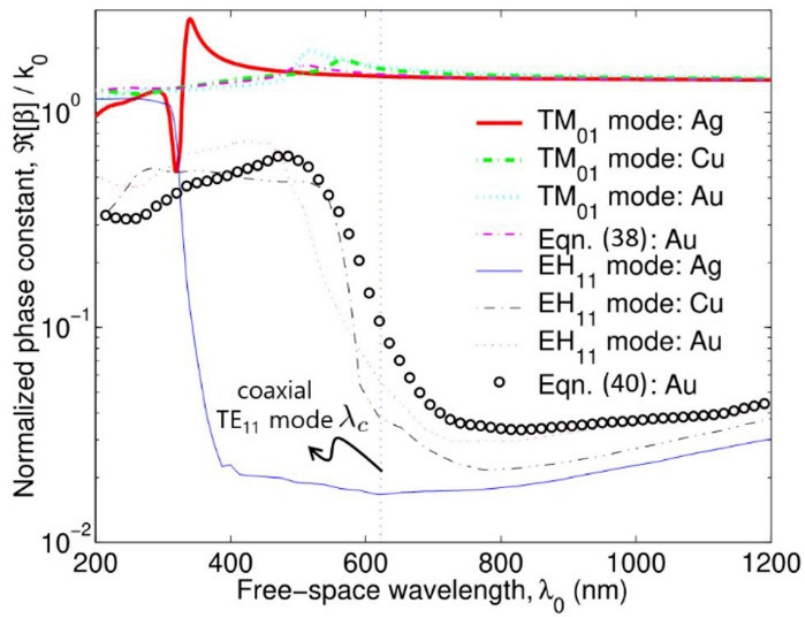

(a)

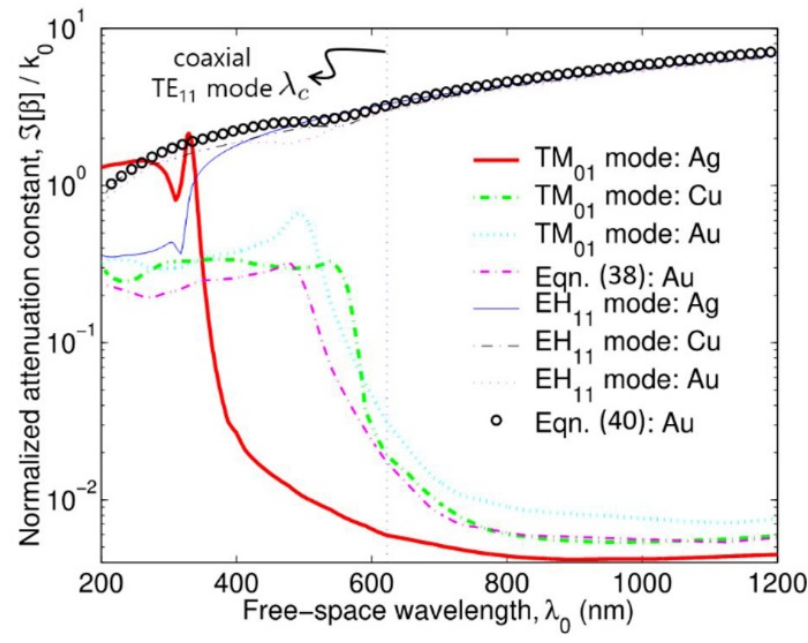

(b)

Fig. 5. Optical behaviors of complex dispersion relations of silver $(\mathrm{Ag})$, copper $(\mathrm{Cu})$, and gold $(\mathrm{Au})$ coaxial waveguides using $m=0$ or $1, a=75 \mathrm{~nm}, b=125 \mathrm{~nm}$, and $d=100 \mathrm{~nm}$, $\mu_{n}=\mu_{0}, \epsilon_{2}=\epsilon_{0}$, and $\epsilon_{1}$ and $\epsilon_{3}\left(\epsilon_{1}=\epsilon_{3}\right)$ are determined by optical dielectric constants of metals: (a) phase constant $\mathfrak{R}[\beta]$ and (b) attenuation constant $\mathfrak{I}[\beta]$. guide composed of real metals, we use optical dielectric constants [12] of silver $(\mathrm{Ag})$, copper $(\mathrm{Cu})$, and gold $(\mathrm{Au})$. In the visible spectrum, the real part of the metal permittivity is usually negative caused by plasma oscillation in the metal. Fig. 5 indicates that Eq. (38) is valid within the optical spectrum and the cutoff or transition wavelength $\left(\lambda_{c}\right)$ can be obtained by Eq. (40). As a result, our approximate solutions obtained from Eqs. (38) and (40) are useful for predicting the optical behaviors of the $\mathrm{TM}_{01}$ and $\mathrm{EH}_{11}$ modes in the nanoscale coaxial waveguide.

\section{CONCLUSION}

Analytical hybrid-mode dispersion relations of the lossy coaxial waveguide are presented based on the mode-matching technique and the vector potential formulations. Precise phase and attenuation constants of the $\mathrm{TM}_{01}, \mathrm{TE}_{01}$, and $\mathrm{EH}_{11}$ modes have been numerically evaluated using the rootsearching algorithm based on Davidenko's method. Approximate but accurate dispersion equations for the $\mathrm{TM}_{0 p}, \mathrm{TE}_{0 p}$, $\mathrm{EH}_{m p}$, and $\mathrm{HE}_{m p}$ modes are also proposed and agree well with more rigorous dispersion relations even for low electric conductivity or negative permittivity. Our dispersion solutions can be applied to the theoretical evaluation of a coaxial calibration kit or the analytical determination of light distribution within the optical coaxial waveguide.

This work was supported by the research fund of Mokwon University in 2019 and conducted at the Center for Electromagnetic Metrology, Korea Re-search Institute of Standards and Science (KRISS), Daejeon, Korea.

\section{REFERENCES}

[1] M. Ibanescu, Y. Fink, S. Fan, E. L. Thomas, and J. D. Joannopoulos, "An all-dielectric coaxial waveguide," Science, vol. 289, no. 5478, pp. 415-419, 2000.

[2] F. I. Baida, A. Belkhir, D. Van Labeke, and O. Lamrous, "Subwavelength metallic coaxial waveguides in the optical range: role of the plasmonic modes," Physical Review $B$, vol. 74, no. 20, article no. 205419, 2006.

[3] B. Rizal, J. M. Merlo, M. J. Burns, T. C. Chiles, and M. J. Naughton, "Nanocoaxes for optical and electronic devices," Analyst, vol. 140, pp. 39-58, 2015.

[4] J. Y. Kwon, J. S. Kang, D. J. Lee, and J. H. Kim, "Analysis of a stepped air line using an air line simulator," MAPAN, vol. 27, no. 2, pp. 105-111, 2012.

[5] C. Cho, J. S. Kang, J. G. Lee, and H. Koo, "Characterization of a $1 \mathrm{~mm}$ (DC to $110 \mathrm{GHz}$ ) calibration kit for 
VNA," Journal of Electromagnetic Engineering and Science, vol. 19, no. 4, pp. 272-278, 2019.

[6] R. DeVore, J. F. Toth, and R. Caldecott, "Dielectric coaxial waveguide," Journal of Applied Physics, vol. 44, no. 10, pp. 4488-4500, 1973.

[7] W. C. Daywitt, "Exact principal mode field for a lossy coaxial line," IEEE Transactions on Microwave Theory and Techniques, vol. 39, no. 8, pp. 1313-1322, 1991.

[8] W. C. Daywitt, "The propagation constant of a lossy coaxial line with a thick outer conductor," IEEE Transactions on Microwave Theory and Techniques, vol. 43, no. 4, pp. 907911, 1995.

[9] H. J. Eom, Electromagnetic Wave Theory for Boundary-Value

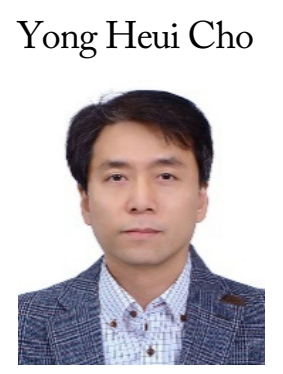

received his B.S. degree in electronics engineering from Kyungpook National University, Daegu, Korea in 1998, and his M.S. degree and Ph.D. in electrical engineering from the Korea Advanced Institute of Science and Technology (KAIST), Daejeon, Korea in 2000 and 2002, respectively. From 2002 to 2003, he was Senior Research Staff with the Electronics and Telecommunications Research Institute (ETRI), Daejeon, Korea. In 2003, he joined the School of Information and Communication Engineering, Mokwon University, Daejeon, Korea, where he is currently a professor. From 2011 to 2012, he was a visiting professor with the Department of Electrical and Computer Engineering, University of Massachusetts Amherst, Amherst, MA, USA. From 2019 to 2020, he was a visiting researcher with the Center for Electromagnetic Metrology, Korea Research Institute of Standards and Science (KRISS), Daejeon, Korea. His current research interests include electromagnetic wave theory and scattering, numerical analysis, design of reflectarrays, and dispersion characteristics of transmission lines.
Problems: An Advanced Course on Analytical Methods. Berlin, Germany: Springer-Verlag, 2004.

[10] H. A. N. Hejase, "On the use of Davidenko's method in complex root search," IEEE Transactions on Microwave Theory and Techniques, vol. 41, no. 1, pp. 141-143, 1993.

[11] Y. H. Cho and D. H. Kwon, "Efficient analytical evaluation of complex dispersion relations of a multiple-row periodic array of magnetodielectric circular cylinders," IEEE Transactions on Antennas and Propagation, vol. 66, no. 5, pp. 2449-2457, 2018.

[12] S. Babar and J. H. Weaver, "Optical constants of $\mathrm{Cu}, \mathrm{Ag}$, and Au revisited," Applied Optics, vol. 54, no. 3, pp. 477481, 2015. 